\section{Fatores de risco maternos associados à acidose fetal}

\section{Maternal risk factors associated with fetal acidosis}

\begin{abstract}
Objective: to assess maternal risk factors associated with fetal acidosis.

Methods: a case-control type study was conducted of 188 neonates, of whom 47 comprised the case group (umbilical arterial $\mathrm{pH} \leq 7.0$ ) and 141 the control (umbilical arterial $p H \geq 7.1 \leq 7.3)$. The study included only single-gestation neonates without congenital malformations. Both maternal and fetal variables were taken into consideration. Statistical analysis involved the calculation of the raw and adjusted Odds Ratio, Student's t-test, the chi-squared test and multivariate analysis using Enter-method non-conditional logistic regression. The level of statistical significance was set at $p<0.05$.

Results: in the case group higher percentages of caesarian sections and pre-term births were observed, involving almost five times as much intensive care and twenty-five times more likelihood of Apgar in the $5^{\text {th }}$ minute $\leq 7$. No association was observed between the groups and fetal presentation, mother's age, history of miscarriage, years of schooling of mother or attendance at prenatal sessions. After multivariate analysis, the only risk factors that remained significant were complications relating to the placenta or the umbilical cord. Deliveries involving complications relating to the placenta or the umbilical cord were three times more likely to involve fetal acidemia.

Conclusions: acidemia among neonates was associated with a higher percentage of caesarians, premature births, a need for intensive care and treatment and an Apgar index of $\leq 7$ in the $5^{\text {th }}$ minute. After multivariate analysis, complications relating to premature displacement of the placenta and the umbilical cord were the only remaining risk factors associated with fetal acidemia.
\end{abstract}

Key words Acidosis, Morbidity, Neonatal, Blood gas analysis
José Mauro Madi 1

Breno Fauth de Araújo 2

Helen Zatti 3

Renato Luís Rombaldi 4

Jucemara Lorencetti 5

Nathalia Oliva Marcon 6

1 Unidade de Ensino Médico de Tocoginecologia. Centro de Ciências da Saúde. Universidade de Caxias do Sul. Av. Prof. Antonio Vignolli, 255. Bairro Petrópolis. Caxias do Sul, RS, Brasil. CEP: 95.001-970. E-mail: jmauromadi@gmail.com

2 Unidade de Ensino Médico de Pediatria. Centro de Ciências da Saúde. Universidade de Caxias do Sul. Caxias do Sul, RS, Brasil. 3 Coordenadora do Serviço de Neonatologista. Unidade de Tratamento Intensivo Neonatal. Hospital Geral/Fundação Universidade de Caxias do Sul. Caxias do Sul, RS, Brasil. 4 Serviço de Ginecologia e Obstetrícia. Hospital Geral/Fundação Universidade de Caxias do Sul. Caxias do Sul, RS, Brasil.

5,6 Universidade de Caxias do Sul. Caxias do Sul, RS, Brasil.

\section{Resumo}

Objetivo: avaliar os fatores de risco maternos associados à acidose fetal.

Métodos: estudo tipo caso-controle composto por 188 recém-nascidos, sendo que 47 compuseram o grupo casos ( $\mathrm{pH}$ de artéria umbilical $\leq 7,0$ ) e 141 os de controles ( $p H$ de artéria umbilical $\geq 7,1 \leq 7,3$ ) nascidos imediatamente após cada caso. Consideraram-se fatores de inclusão: recém-nascidos de gestações únicas e sem malformação congênita. Analisaram-se variáveis maternas e fetais. Foram realizadas a Odds Ratio bruta e ajustada, teste t de Student, teste do qui-quadrado e análise multivariada através da regressão logistica nãocondicional pelo método Enter. Assumiu-se como nível de significância estatística um $p<0,05$.

Resultados: no grupo de casos foi observado maior percentual de cesarianas, de recém-nascidos pré-termo, que apresentaram quase cinco vezes mais necessidade de cuidados intensivos e vinte cinco vezes mais chance de Apgar no $5^{\circ}$ minuto $\leq 7$. Não foram observadas associação entre os grupos e a apresentação fetal, idade materna, história de abortos anteriores, escolaridade materna e frequência ao pré-natal. Após a análise multivariada persistiram como fator de risco complicações relacionadas com a placenta e cordão. Os recémnascidos cujos partos associaram-se a complicações da placenta ou do cordão umbilical apresentaram três vezes mais chance de acidemia fetal.

Conclusões: os recém-nascidos acidóticos estiveram relacionados à maior percentual de cesarianas, de prematuridade, necessidade de cuidados de tratamento intensivo e indice de Apgar $\leq 7$ no $5^{\circ}$ minuto. Após a análise multivariada, persistiram como fator de risco para acidemia fetal as complicações relacionadas ao descolamento prematuro de placenta e cordão umbilical. Palavras-chave Acidose, Morbidade, Neonatal, Gasometria 


\section{Introdução}

A resposta fetal à hipóxia tem sido amplamente estudada, sendo o quadro clínico resultante desse processo denominado de sofrimento fetal. A deficiente troca de gases entre o produto conceptual e o organismo materno promove desequilíbrio no sistema ácido-básico fetal, cuja manifestação bioquímica é representada pela acidose fetal. ${ }^{1}$ Assim, a gasometria realizada no sangue obtido da artéria umbilical tem sido empregada como principal método para aferir a condição de acidose fetal, utilizando os valores do $\mathrm{pH}$ como parâmetro fundamental para esse diagnóstico. ${ }^{2} \mathrm{~A}$ função placentária deficiente, dificultando as trocas gasosas entre o organismo materno e o fetal, é responsável pelo prejuízo na oxigenação fetal. ${ }^{3}$ Como causas de função placentária deficiente citam-se a redução do fluxo sanguíneo materno e da função placentária, e alteração da circulação feto-placentária. O sofrimento fetal decorrente de efeito isquêmico agudo sobre o espaço interviloso ou sobre a própria circulação umbilical origina a acidose fetal que é capaz de produzir alterações neurológicas graves e permanentes, ou mesmo o óbito. 4

Dessa forma, atenção especial tem sido dada à identificação dos recém-nascidos (RN) de alto risco para complicações graves relacionadas à acidose fetal intraparto. 5 Para tanto, tem sido proposta a avaliação do estado ácido-básico fetal, por ocasião do parto, no sangue da artéria umbilical (AU), definindo-se a acidemia fetal patológica (AFP) como um $\mathrm{pH}$ inferior a 7,0 , posto que é neste patamar que ocorre a maior parte das morbidades neonatais. $6-8$

$\mathrm{O} \mathrm{pH}$ da artéria umbilical ( $\mathrm{pH} / \mathrm{AU})$ pode representar importante elemento na avaliação dos cuidados a serem aplicados no período pós-parto imediato. A causa para um baixo índice de Apgar pode ser interpretada como sendo consequência de traumatismos ocasionados no período expulsivo, medicações administradas à gestante, presença de anomalias congênitas ou à descompensação bioquímica por ocasião do nascimento, em fetos já comprometidos durante o período gestacional. ${ }^{9}$ Valores anormais do $\mathrm{pH} / \mathrm{AU}$ permitem ao obstetra e ao neonatólogo analisar criticamente todos os detalhes do trabalho de parto e do parto propriamente dito. ${ }^{9}$

Assim, o estudo realizado visou identificar os principais fatores de risco maternos associados a $\mathrm{RN}$ portadores de AFP.

\section{Métodos}

Estudo do tipo caso-controle, realizado nos Serviços de Ginecologia/Obstetrícia e Unidade de Tratamento Intensivo Neonatal (UTIN) do Hospital Geral da Universidade de Caxias do Sul (HG), mediante o estudo de todos os nascimentos ocorridos no período de Março/1998 a Dezembro/2009.

O HG é hospital público, de nível terciário de atenção, universitário, que atende somente pacientes do Sistema Único de Saúde. O Serviço de Ginecologia e Obstetrícia é referência para gestantes de alto risco da região nordeste do Estado do Rio Grande do Sul, que engloba 50 municípios.

Para fins de estudo, considerou-se como AFP todos os casos cujo $\mathrm{pH} / \mathrm{AU}$ apresentassem valores $\leq 7,0$ (grupo de acidóticos-GAcid). Os controles caracterizaram-se pelos três partos subsequentes ao caso, cujo $\mathrm{pH}$ estivesse $\geq 7,1$ e $\leq 7,3$ (grupo de controles-GContr).

Os dados clínicos e sociodemográficos foram obtidos após revisão dos prontuários médicos no Centro Obstétrico, na Unidade de Internação Obstétrica e na UTIN. As amostras de sangue da AU com vistas à realização da gasometria são colhidas rotineiramente no Centro Obstétrico, por ocasião do nascimento.

Foram excluídas da pesquisa as gestações gemelares, os RN com malformação congênita e os que apresentaram $\mathrm{pH} / \mathrm{AU}$ superior a 7,3, devido à possibilidade de punção inadvertida da veia umbilical. As amostras sanguíneas obtidas da AU foram colhidas imediatamente após o nascimento. A técnica de coleta pode ser assim resumida: antes do primeiro esforço inspiratório do RN, colocação de duas pinças no cordão umbilical próximas ao intróito vaginal (no caso de partos vaginais) ou da histerotomia (no caso de parto cesáreo), e de duas pinças próximas ao coto umbilical. Secção do cordão umbilical entre as pinças proximais e distais. O segmento funicular obtido foi depositado sobre a mesa auxiliar para manipulação, após a ultimação do parto. Foram utilizadas seringas previamente heparinizadas para a punção da AU. Logo após a coleta, a seringa era vedada, cuidando-se para não permitir a entrada de bolhas de ar no seu interior. As amostras sanguíneas foram analisadas em um tempo não superior a trinta minutos, em equipamento AVL OMNI Modular System (Graz, Áustria). 10

As variáveis maternas estudadas foram: altura (em centímetros); idade (em anos); escolaridade (em anos de estudo); ocorrência de abortos prévios à gestação em estudo; realização de pré-natal (sim ou não); apresentação fetal (pélvica ou cefálica); 
presença de síndrome hipertensiva (sim ou não), e/ou diabete melito (sim ou não) e apresentação na carteira de gestante, no prontuário do ambulatório de gravidez de alto risco ou ficha obstétrica, preenchidas por ocasião da internação hospitalar, do registro de diabete melito do tipo 1, 2 ou de diabete melito gestacional;11,12 via de parto (vaginal ou cesáreo); complicações da placenta (descolamento prematuro de placenta) e/ou cordão umbilical (circulares cervicais simples ou múltiplas, apertadas ou não, e nó verdadeiro de cordão). As variáveis fetais foram: percentual de prematuridade (idade gestacional inferior a 37 semanas completas); peso do $\mathrm{RN}$ (em gramas); necessidade de reanimação neonatal, alterações neurológicas (diagnosticadas ao exame físico, tomografia de crânio ou ultrassonografia transfontanelar, por ocasião da alta da UTIN); índice de Apgar no $1^{\circ}$ e $5^{\circ}$ minuto, necessidade de internação em ambiente de intensivismo e/ou óbito e permanência hospitalar (em dias).

Para se avaliar os fatores de risco associados à AFP foi utilizada como variável dependente o pH/AU, obtido por ocasião do nascimento e como variáveis independentes as variáveis maternas.

Os dois grupos foram também comparados em relação à evolução dos RN até a alta hospitalar, utilizando-se como variáveis a necessidade de reanimação neonatal, evolução no berçário, índice de Apgar no $5^{\circ}$ minuto, ocorrência de alteração neurológica na alta hospitalar e/ou óbito.

Para se verificar a presença ou não de associação entre as variáveis independentes e o desfecho estudado estimou-se a Odds Ratio (OR) bruta e ajustada para cada associação de interesse. $\mathrm{Na}$ análise univariada foi aplicado o teste $t$ de Student para as variáveis contínuas (o teste t de Student é utilizado para comparação de médias) e o teste do qui- quadrado para as variáveis dicotômicas. Visando à identificação de possíveis vieses de confusão, foi realizada a análise multivariada através da regressão logística não-condicional, pelo método Enter, utilizado pelo pacote estatístico SPSS para fazer esta análise.

Os critérios de seleção das variáveis para o modelo de regressão logística envolveram a significância estatística $(p<0,05)$ na análise univariada e/ou a relevância clínico-epidemiológia da variável, mediante o conhecimento vigente.

As informações do estudo foram obtidas nos prontuários das gestantes, digitadas e analisadas em um banco de dados do programa SPSS (Statistical Package for the Social Sciences), versão 16.0.

O estudo foi aprovado pelo Comitê de Ensino e Pesquisa da Universidade de Caxias do Sul.

\section{Resultados}

No período citado ocorreram 15.495 nascimentos. Nesse total, foram observados 5.058 estudos gasométricos do sangue arterial do cordão umbilical, sendo que 47 amostras $(0,92 \%)$ apresentaram $\mathrm{pH}$ $\leq 7,0$ (GAcid) e 141 (2,8\%), $\mathrm{pH} \geq 7,1$ e $\leq 7,3$ (GContr).

$\mathrm{O}$ pH/AU médio dos RN incluídos no GAcid e GContr foi de $6,9 \pm 0,12$ e $7,22 \pm 1,75$, respectivamente.

A Tabela 1 apresenta as principais características dos dois grupos. No grupo de RN acidóticos houve predominância da operação cesariana como via de parto $(66 \%$ vs. $42,6 \%)$, de partos pré-termo $(33,3 \%$ vs. $13,9 \%)$, RN com menor peso médio no nascimento $(2846,1 \pm 988 \mathrm{~g}$ vs. $3119,1 \pm 664,3 \mathrm{~g})$ e de índice de Apgar no $1^{\circ}(3,4 \pm 1,0$ vs. $7,6 \pm 1,9)$ e $5^{\circ}(5,9 \pm 1,9$ vs. $9,0 \pm 2,2)$ minutos inferiores.

\section{Tabela 1}

Distribuição das principais características do GAcid e GContr. Hospital Geral de Caxias do Sul, RS, $2002-2007$.

\begin{tabular}{|c|c|c|}
\hline Características & $\begin{array}{l}\text { GAcid* }^{*} \\
(\mathrm{~N}=47)\end{array}$ & $\begin{array}{l}\text { GContr** } \\
(\mathrm{N}=141)\end{array}$ \\
\hline & $\bar{X} \pm D P$ & $\bar{X} \pm D P$ \\
\hline pH médio da artéria umbilical & $6,89 \pm 0,12$ & $7,22 \pm 1,75$ \\
\hline Média da idade materna (anos) & $26,6 \pm 7,2$ & $26,2 \pm 8,1$ \\
\hline Média de consultas no pré-natal & $7,6 \pm 3,5$ & $7,0 \pm 3,7$ \\
\hline Incidência de cesariana (\%) & 66,0 & 42,6 \\
\hline Peso médio do recém-nascido (g) & $2.846,1 \pm 988$ & $3.119,1 \pm 664,3$ \\
\hline Incidência de prematuridade (\%) & 33,3 & 13,9 \\
\hline Apgar médio no $1^{\circ} \min$ & $3,4 \pm 1,0$ & $7,6 \pm 1,9$ \\
\hline Apgar médio no $5^{\circ} \mathrm{min}$ & $5,9 \pm 1,9$ & $9,0 \pm 2,2$ \\
\hline Permanência hospitalar (dias) & $18,5 \pm 2,1$ & $3,0 \pm 0,6$ \\
\hline Mortalidade neonatal (\%) & 23,4 & 0 \\
\hline
\end{tabular}

*GAcid: grupo acidótico; **GContr: grupo controle. 
As variáveis idade materna e número de consultas de pré-natal apresentaram-se de forma semelhante em ambos os grupos.

Os RN incluídos no GAcid permaneceram, em média, 18,5 $\pm 2,1$ dias na UTIN, enquanto os RN do grupo controle permaneceram em média $3,0 \pm 0,6$ dias. A mortalidade foi de $23,4 \%$ de óbitos no grupo com acidose e nenhum óbito no grupo controle.

Na Tabela 2 observou-se que os RN do GAcid apresentaram quase cinco vezes mais chance de evolução desfavorável, com necessidade de UTIN ou evolução para o óbito, e cerca de 25 vezes mais chance de índice de Apgar no $5^{\circ}$ minuto inferior a 7 .

A análise dos fatores de risco relacionados à AFP estão descritas na Tabela 3.
Não se constatou diferença entre os dois grupos estudados em relação à altura da gestante, idade materna, escolaridade, abortos prévios, realização de pré-natal, apresentação fetal, e ocorrência de doença hipertensiva e/ou diabete melito durante a gestação.

Após a análise multivariada persistiram como fator de risco para AFP as complicações relacionadas à placenta e ao cordão, constatando-se cinco casos de descolamento prematuro de placenta e 16 patologias do cordão umbilical (circulares cervicais simples ou múltiplas, apertadas ou não, e nó verdadeiro). Os $\mathrm{RN}$ em cujos partos observaram-se complicações da placenta ou do cordão umbilical apresentaram três vezes mais chance de AFP no momento do nascimento.

Tabela 2

Distribuição dos recém-nascidos, segundo as variáveis de desfecho. Hospital Geral de Caxias do Sul, RS, $2002-2007$.

\begin{tabular}{|c|c|c|c|c|c|c|}
\hline \multirow{2}{*}{ Variáveis } & \multicolumn{2}{|c|}{$\begin{array}{l}\text { GAcid } \\
(\mathrm{N}=47)\end{array}$} & \multicolumn{2}{|c|}{$\begin{array}{l}\text { GContr } \\
(\mathrm{N}=141)\end{array}$} & \multirow{2}{*}{ OR bruta (IC95\%) } & \multirow{2}{*}{ OR ajustada (IC95\%) } \\
\hline & $\mathrm{n}$ & $\%$ & $\mathrm{n}$ & $\%$ & & \\
\hline \multicolumn{7}{|c|}{ Reanimação neonatal } \\
\hline Sim & 19 & 40,4 & 7 & 5,0 & $12,9(4,9-33,8)$ & $1,7(0,4-6,4)$ \\
\hline Não** & 28 & 59,6 & 134 & 95,0 & 1 & 1 \\
\hline \multicolumn{7}{|l|}{ Apgar $5^{\circ} \mathrm{min}^{*}$} \\
\hline$<7$ & 22 & 51,2 & 2 & 1,4 & $71,7(15,7-327,6)$ & $24,8(4,4-140,9)$ \\
\hline$\geq 7^{* *}$ & 21 & 48,8 & 137 & 98,6 & 1 & 1 \\
\hline \multicolumn{7}{|c|}{ Evolução no berçário } \\
\hline UTI ou óbito & 33 & 70,2 & 18 & 12,8 & $16,1(7,2-35,7)$ & $4,7(1,66-13,1)$ \\
\hline Alta** & 14 & 29,8 & 123 & 87,2 & 1 & 1 \\
\hline \multicolumn{7}{|c|}{ Alteração neurológica*** } \\
\hline Sim & 8 & 17,0 & 2 & 1,4 & $14,2(2,9-69,8)$ & $0,9(0,1-8,3)$ \\
\hline Não** & 39 & 83,0 & 139 & 98,6 & 1 & 1 \\
\hline
\end{tabular}

* Eventuais diferenças são decorrentes de dados sem informação precisa; ** Referência; *** Inclui alteração ao exame neurológico, tomografia de crânio ou ultrassonografia transfontanelar por ocasião da alta hospitalar; GAcid= grupo acidótico; GContr= grupo controle. 
Distribuição dos nascidos vivos segundo variáveis maternas e da gestação, Hospital Geral de Caxias do Sul, RS, 20022007.

\begin{tabular}{|c|c|c|c|c|c|c|}
\hline \multirow{2}{*}{ Variáveis } & \multicolumn{2}{|c|}{$\begin{array}{l}\text { GAcid } \\
(\mathrm{N}=47)\end{array}$} & \multicolumn{2}{|c|}{$\begin{array}{l}\text { GContr } \\
(\mathrm{N}=141)\end{array}$} & \multirow{2}{*}{ OR bruta (IC95\%) } & \multirow{2}{*}{ OR ajustada (IC95\%) } \\
\hline & $\mathrm{n}$ & $\%$ & $\mathrm{n}$ & $\%$ & & \\
\hline Altura da gestante $(\mathrm{cm})$ & & & & & & $2,9(0,9-9,5)$ \\
\hline$<150$ & 7 & 20,6 & 8 & 6,9 & $3,5(1,7-10,5)$ & \\
\hline$\geq 150^{* *}$ & 27 & 79,4 & 108 & 93,1 & 1 & \\
\hline \multicolumn{7}{|l|}{ Idade materna (anos) } \\
\hline$>34$ & 6 & 17,1 & 20 & 19,0 & $0,9(0,3-2,4)$ & \\
\hline $20-34^{* *}$ & 29 & 82,9 & 85 & 81,0 & 1 & \\
\hline \multicolumn{7}{|l|}{ Escolaridade } \\
\hline Até $1^{\circ} \mathrm{grau}$ incompleto & 27 & 64,3 & 68 & 52,3 & $1,6(0,8-3,7)$ & \\
\hline$\geq 1^{\circ}$ grau completo** & 15 & 35,7 & 62 & 47,7 & 1 & \\
\hline \multicolumn{7}{|l|}{ Abortos prévios } \\
\hline Sim & 8 & 17,0 & 18 & 12,8 & $1,4(0,6-3,5)$ & \\
\hline Não** & 39 & 83,0 & 123 & 87,2 & 1 & \\
\hline \multicolumn{7}{|l|}{ Pré-natal } \\
\hline Não & 0 & - & 8 & 5,7 & & \\
\hline $\operatorname{Sim}^{* *}$ & 47 & 100,0 & 133 & 94,3 & & \\
\hline \multicolumn{7}{|l|}{ Apresentação fetal } \\
\hline Pélvica & 6 & 12,8 & 17 & 12,1 & $1,1(0,4-2,9)$ & \\
\hline Cefálica** & 41 & 87,2 & 124 & 87,9 & 1 & \\
\hline \multicolumn{7}{|l|}{ Hipertensão/Diabete } \\
\hline Sim & 15 & 31,9 & 24 & 17,0 & $2,2(1,1-4,8)$ & $1,9(0,7-4,9)$ \\
\hline Não** & 32 & 68,1 & 117 & 83,0 & 1 & 1 \\
\hline \multirow{2}{*}{\multicolumn{7}{|c|}{$\begin{array}{l}\text { Complicações da placenta } \\
\text { e/ou cordão umbilical\# }\end{array}$}} \\
\hline & & & & & & \\
\hline Sim & 21 & 44,7 & 28 & 20,0 & $3,2(1,6-6,5)$ & $3,3(1,5-7,7)$ \\
\hline Não** & 26 & 55,3 & 112 & 80,0 & 1 & 1 \\
\hline
\end{tabular}

*As diferenças são decorrentes de dados sem informação; **Referência; GAcid= grupo acidótico; GContr= grupo controle; \# Cinco casos de descolamento prematuro de placenta no GAcid e um caso no GContr.

\section{Discussão}

No presente estudo metade dos RN com AFP apresentou Apgar no $5^{\circ}$ minuto inferior a sete e $70 \%$ necessitaram de tratamento intensivo neonatal ou evoluíram para o óbito, demonstrando as graves consequências deste tipo de acidose. Estudos anteriores mostram que neonatos que apresentam AFP por ocasião do nascimento podem apresentar maior risco de morbidade e resultados adversos no período neonatal imediato.5,13-15 Entretanto, é importante enfatizar que a condição citada tem incidência baixa nos vários relatos da literatura. 16,17

Outra variável de desfecho pesquisada foi a alteração neurológica no RN no momento da alta, sendo incluída nesta variável qualquer alteração identificada no exame neurológico, tomografia de crânio ou ultrassonografia transfontanelar. O GAcid apresentou $17 \%$ de $\mathrm{RN}$ com alterações neurológicas e o GContr, $1,4 \%$. Embora a análise univariada tenha mostrado diferença entre os grupos, esta diferença desapareceu após a análise multivariada, provavelmente devido ao pequeno número da amostra. Estudos têm mostrado a relação entre o índice de Apgar no $5^{\circ}$ minuto inferior a sete e morbidades neonatais, em RN com $\mathrm{pH} / \mathrm{AU}<7,0$. Smith et al. 18 sugerem que a combinação de marcadores pós-natais de alto risco, como o índice de Apgar no $5^{\circ}$ minuto inferior a sete, a necessidade de entubação em sala de parto e a associação com acidemia fetal grave, 
facilitaria a identificação dos $\mathrm{RN}$ a termo com maior risco de desenvolver convulsões secundárias à asfixia perinatal. Locatelli et al. ${ }^{19}$ apontam a associação entre a acidose fetal patológica e crises convulsivas neonatais. Entretanto, a relação entre asfixia fetal e anormalidades neurológicas em longo prazo, como a paralisia cerebral, ainda não está bem estabelecida. Borruto et al. ${ }^{20}$ referiram que somente $10 \%$ a $15 \%$ dos casos de paralisia cerebral são de fato devido à asfixia perinatal.

Estudos que avaliaram a relação entre a acidose fetal patológica e resultados neurológicos em $\mathrm{RN}$ a termo e pré-termo evidenciaram aumento na ocorrência de hemorragia intraventricular grave em prematuros, e também aumento das convulsões em prematuros e em $\mathrm{RN}$ a termo que foram submetidos à acidose no nascimento. Nenhum efeito significativo da acidemia pode ser demonstrado em longo prazo. ${ }^{14,21}$

Avaliando os fatores de risco relacionados à acidemia no nascimento, observou-se uma associação estatisticamente significante com as patologias da placenta e cordão umbilical, diferença que persistiu mesmo após o controle dos fatores de confusão. Resultados semelhantes foram descritos por Victory et al.,22 que evidenciaram incidência de $0,4 \% \mathrm{de} \mathrm{pH} / \mathrm{AU}$ igual ou inferior a 7,0 em uma amostra de 8992 RN. Nesse estudo, os fatores de risco associados à acidose fetal foram o descolamento de placenta e as complicações de cordão umbilical.22

\section{Referências}

1. Goldaber KG, Gilstrap LC 3rd. Correlations between obstetric clinical events and umbilical cord blood acid-base and blood gas values. Clin Obstet Gynecol. 1993; 36: 4759.

2. Francisco RPV, Yamamoto RM, Miyadahira S, Cunha CL, Zugaib M. Correlação entre testes para avaliação da vitalidade fetal, $\mathrm{pH}$ da artéria umbilical e os resultados neonatais em gestações de alto risco. Rev Bras Ginecol Obstet. 2000; 22: 503-10.

3. Nomura RMY, Francisco RPV, Miyadahira KSS, Zugaib M. Centralização da circulação fetal em gestações de alto risco: avaliação da vitalidade fetal e resultados perinatais. Rev Bras Ginecol Obstet. 2001; 23: 137-43.

4. Mendez-Bauer C, Shekarloo A, Cook V, Freese U Treatment of acute intrapartum fetal distress by b2-sympathomimetics. Am J Obstet Gynecol. 1987; 156: 638-43.

5. Blickstein I, Green T. Umbilical cord blood gases. Clin Perinatol. 2007; 34: 451-9.

6. Andreani M, Locatelli A, Assi F, Consonni S, Malguzzi S,
Quanto à apresentação fetal, a despeito desta variável não apresentar significância estatística no estudo, Borruto et al. 20 e Victory et al. 22 realizaram estudos com objetivos de identificar fatores de risco para acidemia no nascimento e apontaram a apresentação pélvica e a circular de cordão como variáveis independentemente associadas. Borruto et al.,20 estudando $249 \mathrm{RN}$ com acidemia e $249 \mathrm{RN}$ como grupo controle, observaram uma associação entre a acidemia e a apresentação fetal pélvica, administração de ocitocina e meperidina, circular de cordão e RN do sexo masculino.

Em relação à altura das gestantes, não houve diferença após a análise multivariada para controle dos fatores de confusão. Entretanto, mulheres com altura inferior a $150 \mathrm{~cm}$, quando comparadas as de altura superior a esse parâmetro, apresentaram 2,5 vezes mais risco de parto cesáreo por alteração da vitalidade fetal intraparto, com a consequente acidose fetal, conforme relatam Merchant et al.17

Assim, o pH/AU representa o mais sensível dos marcadores de hipoxemia fetal intraparto. A AFP consequente ao sofrimento fetal, indicou com maior frequência o nascimento de $\mathrm{RN}$ por cesariana. Observou-se uma associação entre a AFP e as variáveis prematuridade, índice de Apgar $\leq 7$, descolamento prematuro de placenta e patologias de cordão umbilical, determinando um maior número de RN necessitados de cuidados de tratamento intensivo.

Paterlini G, Ghidini, A. Predictors of umbilical artery acidosis in preterm delivery. Am J Obstet Gynecol. 2007; 197: 303. e1-5.

7. Salhab WA, Perlman JM. Severe fetal acidemia and subsequent neonatal encephalopathy in the larger premature infant. Pediatr Neurol. 2005; 32: 25-9.

8. ACOG Committee Opinion No. 348, November 2006. Umbilical cord blood gas and acid-base analysis. Obstet Gynecol. 2006; 108: 1319-22.

9. Zupan Simunek V. Definition of intrapartum asphyxia and effects on outcome. J Gynecol Obstet Biol Reprod 2008;37 Suppl 1:S7-15.

10. Madi J, Morais E, Festugatto J, Treméa C, Rigotto M. Efeito da heparina liofilizada e da líquida sobre a medida do $\mathrm{pH}$ do sangue do cordão umbilical. Rev Bras Ginecol Obstet. 2003; 25: 371-3

11. [No authors]. Report of the National High Blood Pressure Education Program Working Group on High Blood Pressure in Pregnancy. Am J Obstet Gynecol. 2000; 183: S1-S22. 
12. Expert Committee on the Diagnosis and Classification of Diabetes Mellitus Report of the Expert Committee on the Diagnosis and Classification of Diabetes Mellitus. Diab Care 2004;27:S5-S20.14

13. Modarressnejad V. Umbilical cord blood $\mathrm{pH}$ and risk factors for acidaemia in neonates in Kerman. East Mediterr Health J. 2005; 11: 96-101

14. De Franco S, Esposito S, Rossaro D, Bona G, Ferrero F. Risk factors in newborns with severe acidosis at birth. Panminerva Med. 2007; 49: 17-9.

15. Van den Berg PP, Nelen WL, Jongsma HW, Nijland R, Kollee LA, Nijhuis JG, Eskes TK informar nome de todos autores em vez do et al. Neonatal complications in newborns with an umbilical artery $\mathrm{pH}<7.00$. Am J Obstet Gynecol. 1996; 175: 1152-7.

16. Sehdev HM, Stamilio DM, Macones GA, Graham E, Morgan MA. Predictive factors for neonatal morbidity in neonates with an umbilical arterial cord $\mathrm{pH}$ less than 7.00 Am J Obstet Gynecol. 1997; 177: 1030-4.

17. Merchant KM, Villar J, Kestler E. Maternal height and newborn size relative to risk of intrapartum caesarean delivery and perinatal distress. BJOG 2001;108:689-96.
18. Smith EM, Johnson SR, Cripe T, Perlman S, McGuinness G, Jiang D, Cripe L, Turek LP. Perinatal transmission and maternal risks of human papillomavirus infection. Cancer Detect Prev. 1995; 19: 196-205

19. Locatelli A, Incerti M, Ghidini A, Greco M, Villa E, Paterlini G. Factors associated with umbilical artery acidemia in term infants with low Apgar scores at 5 min. Eur J Obstet Gynecol Reprod Biol. 2008; 139: 146-50.

20. Borruto F, Comparetto C, Treisser A. Prevention of cerebral palsy during labour: role of foetal lactate. Arch Gynecol Obstet. 2008; 278: 17-22.

21. Lavrijsen SW, Uiterwaal CS, Stigter RH, de Vries LS, Visser GH, Groenendaal F. Severe umbilical cord acidemia and neurological outcome in preterm and full-term neonates. Biol Neonate. 2005; 88: 27-34

22. Victory R, Penava D, Da Silva O, Natale R, Richardson B. Umbilical cord $\mathrm{pH}$ and base excess values in relation to adverse outcome events for infants delivering at term. Am J Obstet Gynecol. 2004; 191: 2021-8.

Recebido em 25 de março de 2009

Versão final apresentada em 19 de julho de 2010

Aprovado em 30 de julho de 2010 